\section{MIT goes platinum}

\author{
By Kai-Jye Lou, Staff Writer
}

Researchers at the Massachusetts Institute of Technology have synthesized mitaplatin, a fusion between cisplatin and dichloroacetate that could have a better therapeutic window than cisplatin and could treat cancers that are resistant to the platinum-based chemotherapeutic. ${ }^{1}$ The group now is running in vivo experiments hoping to replicate in vitro results in which mitaplatin showed synergistic improvements in selectivity for cancer cells compared with each of its components delivered separately.

In the intracellular environment, cisplatin promotes cell death by entering the nucleus and disrupting the activity of DNA. However, the nonspecificity of cisplatin's mechanisms also contributes to dose-limiting side effects such as kidney damage, nerve damage and hearing loss. In addition, cancer cells can acquire resistance to cisplatin by a variety of mechanisms including a defective cell death response.

To address both the toxicity and resistance issues, researchers at MIT hypothesized that a chemotherapeutic that attacks both the nucleus and mitochondria-based metabolism pathways of cancer cells could be more effective. Indeed, multiple studies have already suggested that targeting cancer-specific pathways in mitochondria could circumvent their resistance to apoptosis. ${ }^{2}$

The MIT group decided to engineer a fusion prodrug that would pair cisplatin with dichloroacetate (DCA), a generic inhibitor of mitochondrial pyruvate dehydrogenase kinase (PDK). PDK is highly expressed in cancer cells.

In 2007, a group at the University of Alberta showed that DCA selectively reverses apoptosis resistance in cancer cells without significantly affecting normal cells. ${ }^{3}$ That university is running a Phase II study of DCA for anaplastic astrocytoma and glioblastoma.

The MIT group designed a cisplatin prodrug by fusing cisplatin with two units of DCA. The resulting compound, dubbed mitaplatin, "takes advantage of two different mechanisms of action," said Shanta Dhar, a postdoctoral fellow in the Department of Chemistry at MIT. "Upon entering the cell, mitaplatin is reduced to its cisplatin component, which attacks DNA in the nucleus, and the DCA components, which alter the way that mitochondria function."

In multiple panels of human cell lines, mitaplatin destroyed cancer cells while showing low toxicity against normal fibroblasts. In contrast, cisplatin alone or a nonfusion mixture of cisplatin and DCA indiscriminately killed cancer cells and normal fibroblasts. DCA alone had low toxicity against normal fibroblasts but little potency against cancer cells compared with mitaplatin or cisplatin alone.

Although many platinum-based prodrugs have shown lower cytotoxicity than cisplatin, they also are less potent against cancer cells. In contrast, mitaplatin demonstrated potency comparable to that of cisplatin.

Results were published in the Proceedings of the National Academy of Sciences.

"The interesting and unexpected result was that when we evaluated mitaplatin in cell culture, it selectively killed cancer cells, whereas cisplatin indiscriminately killed both cancer cells and normal fibroblasts," said Stephen Lippard, senior author on the paper and a professor of chemistry at MIT. "One might think that if you added a combination of cisplatin and DCA you would get an effect similar to that of mitaplatin, but you don't. Combined treatment with cisplatin and DCA still indiscriminately killed both cell types."

"Since mitaplatin appears to be less toxic toward normal cells, you could potentially deliver higher doses to increase efficacy and overcome issues like resistance," said Dhar, who was lead author on the paper.

"The experiments show mitaplatin has essentially the same activity as cisplatin and is far superior to DCA used alone," said Kosta Steliou, chairman and CEO of PhenoMatriX Inc. and a professor at the Boston University School of Medicine Cancer Research Center. "An anticancer drug that selectively kills cancer cells, while sparing normal cells in the process, is desperately needed. If mitaplatin has this capacity in vivo, it could become a breakthrough treatment for

cancer."

PhenoMatriX has multiple small molecules in preclinical development to treat cancer. Steliou did not elaborate on the chemical class and mechanisms of the company's lead compounds, but he holds a patent covering a class of cisplatin derivatives called mitoplatins, which can target to mitochondrial DNA via the carnitine-acylcarnitine translocase system.

\section{Recipe for synergy}

Although the mechanisms underlying mitaplatin's synergistic effects are not yet clear, Dhar said they could be related to how the molecule is taken up into the cell.

"Mitaplatin uses a single uptake mechanism to bring both anticancer agents into the cell at the same time," she told SciBX. "When DCA and cisplatin are given separately, their uptake mechanisms are different, so the two compounds may not be getting to the right parts of the cell at the right time to produce the synergistic effects that we see with mitaplatin."

Lippard speculated that mitaplatin's increased selectivity may be due to potentiation of its cisplatin payload through altering mitochondrial function in cancer cells. In fact, analytical studies in the PNAS paper showed that mitaplatin accumulated to comparable levels in both types of cells.

Steliou noted that the mitaplatin data run counter to results from previous attempts to fuse cisplatin with a separate cancer therapeutic. 
"Past experience with prodrugs designed to discharge two or more independently active components has generally not yielded a therapeutic advantage over concurrent administration of the drugs separately," he told SciBX.

Steliou pointed to one such example reported in a 2009 study from the Royal College of Surgeons in Ireland, which described a cisplatin conjugate that released cisplatin concomitantly with a histone deacetylase (HDAC) inhibitor once inside the cell. ${ }^{4}$

The mitaplatin results reported in PNAS appear to buck this trend.

According to Steliou, the increased apoptotic efficacy apparently added by DCA, as well as mitaplatin's selective preference for targeting tumor cells, "seems to go against the historical trend and also the lack of any observed synergistic activity" between DCA and cisplatin, which was reported in a 2008 study by a group at The Ohio State University. ${ }^{5}$

The Ohio study showed that cisplatin plus DCA did not produce an additive effect on apoptosis in two human non-small cell lung cancer (NSCLC) cell lines.

\section{Getting the right mix}

The MIT group next hopes to show that the synergistic effects of the mitaplatin components seen in cell culture translate to animal models.

"This work represents an opportunity to see if targeting cancer metabolism can boost the efficacy of platinum chemotherapeutics," said Matthew Vander Heiden, an assistant professor at the David H. Koch Institute for Integrative Cancer Research at MIT.

Vander Heiden did note that the use of a single molecule could have trade-offs.

"If you give two compounds separately, you would have more control over the dosing of each individual compound, which could help address toxicity issues if they arise," he said. "However, by simultaneously delivering two active compounds as a single agent, you can get both into the cell at the same time. This simplifies things from a drug development perspective as you won't have to separately evaluate each compound in the clinic and deal with the delivery of two compounds."
Peter Sadler, head of the Department of Chemistry at the University of Warwick, said the prodrug strategy makes mitaplatin inherently less reactive than cisplatin, which potentially could become an issue. "The problem is that such compounds would need to rely on reducing agents in the body to activate the drug. Future work should include studies to investigate whether mitaplatin is active in the body and not merely against cancer cells in culture," he said.

of Technology

Lippard said his group is evaluating the toxicity, pharmacokinetics and efficacy of mitaplatin in animal models.

According to Lippard and Dhar, preliminary results from maximum tolerated dose studies indicate that the improved selectivity of mitaplatin seen in vitro may be preserved in vivo, as it can be tolerated at much higher doses than cisplatin.

MIT has filed for a patent covering the composition of matter for mitaplatin and its methods of use. The compound is available for licensing.

Lou, K.-J. SciBX 3(3); doi:10.1038/scibx.2010.74

Published online Jan. 21, 2010

REFERENCES

1. Dhar, S. \& Lippard, S.J. Proc. Natl. Acad. Sci. USA; published online Dec. 7, 2009;

doi:10.1073/pnas.0912276106

Contact: Stephen J. Lippard, Massachusetts Institute of Technology, Cambridge, Mass.

e-mail: lippard@mit.edu

2. Don, A.S. \& Hogg, P.J. Trends Mol. Med. 10, 372-378 (2004)

3. Bonnet, S. et al. Cancer Cell 11, 37-51 (2007)

4. Griffith, D. et al. Chem. Commun. 44, 6735 -6737 (2009)

5. Otterson, G.A. et al. J. Clin. Oncol. 26,14637 (2008)

COMPANIES AND INSTITUTIONS MENTIONED

Boston University School of Medicine, Boston, Mass.

Massachusetts Institute of Technology, Cambridge, Mass.

The Ohio State University, Columbus, Ohio

PhenoMatriX Inc., Boston, Mass.

Royal College of Surgeons in Ireland, Dublin, Ireland

University of Alberta, Edmonton, Alberta, Canada

University of Warwick, Coventry, U.K. 